\title{
1st Egyptian International Neuroepidemiology Conference
}

Luxor, Egypt, February 10-13, 2009

\section{Selected Abstracts}

Chairperson

Hamdy N. El-Tallawy, Assiut

Guest Editors

Jeffrey L. Cummings, Los Angeles, Calif.

Valery Feigin, Auckland

Jun Kimura, lowa City, lowa

Giovanni Savettieri, Palermo

Tatsuya Mima, Kyoto

\section{Contents}

Session I. Epilepsy

Abstracts 1-3

Session II. CVS

Abstracts 4-7

Session III. Dementia

Abstracts 8-9

Session IV. Extrapyramidal Disorders

Abstracts 10-14

Session V. Miscellaneous

Abstracts 15-24 


\section{Session I. Epilepsy}

1

\section{Epidemiology of Epilepsy in Al-Kharga District-New Valley (Egypt)}

Hamdy N. El-Tallawya, Wafaa M. Farghalya, Ghaydaa A. Shehata', Noha M. Abo-Elfetoh", Nabil A. Metwally', Tarek A. Rageha, Ahmed Hegazy

aDepartment of Neurology and Psychiatry, Assiut University and bel Azhr University- Assuit Branch, Assiut, Egypt

Background: Epilepsy is the most common serious neurological disorder (sandy 2003). WHO estimates that 8 people per 1000 world wide have epilepsy (Sander 2003, kwan and Sander 2004). The prevalence of epilepsy in developing countries is usually higher than in developed countries (Preux and, Druet - Cabanac 2005).

Aim of the Work: To study epidemiology of epilepsy in Al-Kharga District (New Valley).

Subjects and Methods: This is a cross sectional study on the total population $(62,583)$ of Al-kharga District (New Valley), with $51.4 \%$ males and $48.6 \%$ females. The study was carried out through 3 stages from April $1^{\text {st }} 2005$ to July $31^{\text {st }} 2008$. All population was screened through a door to door study (including every door), in both urban and rural communities by 3 specialists of Neurology, and 15 female social workers to collect demographic data.

All suspected epileptic patients identified by the screening questionnaire, were subjected to detailed history taking, full clinical examination, EEG, and IQ. MRI, video-monitoring and estimation of serum level of AED, were done when indicated.

Results: Four hundred and thirty seven patients (437) with epilepsy (not including febrile convulsions) were identified with a lifetime prevalence rate of $6.98 / 1000(95 \%$ CI $6.33-7.64)$, with a slightly higher rate for females (7.3/1000) than males (6.7/1000), and a slightly higher rate for rural (7.2/1000) than urban (6.9/1000) communities. A total incidence rate of epilepsy during Jun $1^{\text {st }} 2007$ to May $31^{\text {st }} 2008$ was $43.14 / 100.000$ population $(95 \%$ CI $26.87-59.41)$ with a $\mathrm{U}$ - shaped figure of age-specific incidence rate, with 2 Peaks of incidence during infancy $(0-<2$ years $) 172.0 / 100.000$ and during elderly personnel $(\geq 60) 101.6 / 100.000$ population. A $74.3 \%$ of epileptic Patients $(n=326)$, still have active epilepsy, of whom $37.4 \%(n=122)$ did not receive any anti-epileptic medication. Another 70 Patients with active epilepsy were not appropriately treated (having irregular therapy or sub therapeutic doses). This yields a treatment gap of $58.9 \%$ - $(\mathrm{n}=192 / 326)$.
2

\section{Epidemiology of Uncontrolled Epilepsy in Al-Kharga District-New Valley (Egypt)}

Wafaa M.A. Farghalya, Hamdy N. El-Tallawya, Tarek A. Rageha, Eman M. Mohamed ${ }^{\mathrm{b}}$, N. Abdel Hakeem M', Ghaydaa A. Shehataa, Noha M. Abo-Elfetoha, Mohamed A. Abdelhamed ${ }^{\text {a }}$

aDepartment of Neurology \& Psychiatry, Assiut University, ${ }^{b}$ Department of Public Health \& Community Medicine, Assiut University, Assiut, Egypt, 'Alazhar University, Assiut Branch

Background: Uncontrolled epilepsy despite, state-of-the art, medical treatment continues to be a major clinical problem (Brodie, 2005).

Objectives: Identification of true cases of intractable epilepsy, as well as causes and predictors of intractability to allow physicians to consider them earlier for treatment with recent medications and other treatment strategies

Methods: This is a cross sectional study carried out through 3 stages from April $1^{\text {st }} 2005$ to July $31^{\text {st }} 2008$. Total population of 62,583 persons were screened through door to door (every door) by three specialists of neurology and 15 female social workers. All cases of epilepsy were subjected to complete history taking, meticulous examination, conventional EEG, Stanford-Binet intelligence scale. Cases received AEDs in the last 6 months and still have active seizures were considered uncontrolled (Ohtsuka et al, 2000). They were subjected to drug monitoring, video monitoring EEG, MRI and quality of life assessment (QOLIE-31). 50 patients with controlled epilepsy (2 years seizure free), were used for statistical analysis and comparison with true intractable patients.

Results: We identified 437 epileptic patients with prevalence rate $6.98 / 1000$, out of whom, $30.6 \%(n=134 / 437)$ were uncontrolled, with a prevalence rate of 2.1/1000. Noncompliance and sub-therapeutic prescriptions were determined among $52.2 \%(n=70 / 134)$, while $47.8 \%(n=64 / 134)$ of patients were compliant on appropriate treatment. Video monitoring, EEG of compliant uncontrolled patients demonstrated that $78.1 \%(n=50 / 64)$ patients were truly epileptic, while $21.9 \%(n=14 / 64)$ were psychogenic, having non epileptic seizures. Possible etiology of intractable epilepsy was determined among $42 \%$ of cases (Remote symptomatic), while unknown causes were reported among $58 \%$ of cases (idiopathic \& cryptogenic). Perinatal insults were the commonest causes $(23.8 \%)$ of symptomatic uncontrolled epilepsy among children $(<18$ yrs), while cerebrovascular stroke $(20.7 \%)$ was the commonest cause of among adulthood and elderly patients ( $\geq 18$ yrs). Serial fits, status epilepticus, high seizure frequency, multiple seizure type, partial seizure, myoclonic seizures, and severity of EEG epileptic changes are predictors of intractability.

\begin{tabular}{ll}
\hline KARGER & (c) 2009 S. Karger AG, Basel \\
Fax +4161306 1234-5350/09/0323-0242\$26.00/0 \\
$\begin{array}{l}\text { E-Mail karger@karger.ch } \\
\text { www.karger.com }\end{array}$ & $\begin{array}{l}\text { Accessible online at: } \\
\text { www.karger.com/ned }\end{array}$
\end{tabular}




\section{3 \\ Prevalence and Risk Factors of Epilepsy in Egyptian School Children}

Fathi M. Afifi, M. Safwat I., M.A. Al Shaar, Y.D. Shora,

M. Esam, M. Yousof

Faculty of Medicine, Neurology Department, Al Azhar

University, Cairo, Egypt

Background: Epidemiological study is important to identify those who are at risk of epilepsy, and improve the understanding of the causes, and mechanisms of epilepsy.

Subjects and Methods: The present study was conducted on school age children of 6-15 years in Cairo (urban) and Kafr-El-Sheikh (rural),13 primary and preparatory schools were visited, 5221 children were submitted to an open interview.

Results: Twenty seven cases had active epilepsy, gave prevalence rate $(5,17 / 1000)(4,58 / 1000)$ in Cairo, and $(5,59 / 1000)$ in Kafr-El-Shaikh

\section{Session II. CVS}

\section{4}

\section{Epidemiology of Non-Fatal Cerebrovascular Stroke (CVS) in Al-Kharga District-New Valley (Egypt)}

Wafaa M. Farghalya, Hamdy N. El Tallawya, N. Abdel Hakeem ${ }^{b}$, M. Ghaydaa A. Shehata', Abo-Elfetoh Na,

Tarek A. Rageha, Ahmad A.M. Hegazy', Essam M. El Mesalhy ${ }^{\mathrm{b}}$, Bastawy M. El Fawa/

aDepartment of Neurology and Psychiatry, Assiut University and ${ }^{\mathrm{b}} \mathrm{Al}$ Azhar University (Assiut Branch), Assiut, Egypt

Background: Stroke is a medical emergency and can cause permanent neurological damage, complications, adult disability and death (Feigin, 2005). Stroke is currently the second leading cause of death in the western world, ranking after heart disease and before cancer and causes $10 \%$ of deaths world wide (WHO, 2004).

Aim: To determine epidemiology of non fatal CVS in Al- kharga district- New Valley.

Methods: This is a cross sectional study, carried out through 3stages from April $1^{\text {st }}, 2005$ to July $31^{\text {st }}, 2008$. Total population 62,583 were screened through a door to door (every door) study in both urban and rural communities by 3 specialists of neurology and 15 females social workers (for demographic data). All probable subjects with stroke were subjected to a full clinical examination, CT\& or MRI brain, fasting blood sugar, lipogram, serum uric acid, CBC, blood urea, and serum creatinine, as well as Barthel Index and Scandinavian Stroke Scale to evaluate stroke severity. Carotid Doppler, Echocardiography and thyroid function tests were done for selected cases.

Results: Cerebrovascular stroke was recorded among 351 subjects, out of which 156 subjects were recorded during one year (from January $1^{\text {st }}$ to December $31^{\text {st }} 2007$ ), yielding a total prevalence rate of 5.6 $/ 1000$ population and an incidence rates of $2.5 / 1000$. There was a highly signifi- cant differences between urban (5.8/1000 and 2.6/1000 for prevalence and incidence respectively) and rural communities (5.2/1000 and 2.3/1000 for prevalence and incidence respectively). Prevalence and incidence were significantly higher among males (6.1/1000 and 2.7/1000) than females $(5.1 / 1000$ and $2.3 / 1000)(\mathrm{P}$. value $=0.002)$ with the same frequency of distribution in urban and rural communities. Prevalence and incidence rates increased steadily with advancing age to be 53.8/1000 (prevalence) and 23.1/1000 (incidence) among aged persons ( 60 years). The highest prevalence and incidence rates were 4.2/1000 and 1.7/1000 for thrombosis stroke representing $74.4 \%$ of total strokes, whereas the least prevalence and incidence were for those of subarachnoid hemorrhage $(0.03 / 1000$ and $0.02 / 1000)$ representing $0.6 \%$ of total strokes.

5 Epidemiology of Post-Stroke Depression in
Al-Kharga District-New Valley (Egypt)

Hamdy N. El-Tallawya, Hossam Khalifaa, Yaser M.B. El-Serogy', Ghydaa A. Shehataa', Ekram I. Mohamed ${ }^{\mathrm{b}}$

aNeurology and Psychiatry, Faculty of Medicine, Assiut University, bPsychiatric Nursing and Mental Health, Faculty of Nursing, Assiut University, Assiut, Egypt

Background: Depression in survivors of stroke is both common and clinically relevant. It is associated with excess suffering, handicap, suicidal ideation and mortality, and it hampers rehabilitation.

Aim: To assess the prevalence of depression among stroke patients in Al-kharga District, New Valley.

Methods: This is a cross-sectional study that was carried out through 3 stages from April $1^{\text {st }} 2005$ to July $31^{\text {st }} 2008$. The total population 62,583 was screened through door to door (every door) study by 3 specialists of Neurology, and 15 female social workers. A total 301 cases of stroke were diagnosed on clinical and radiological basis. 271 cases were assessed by two specialists of psychiatry at the General Hospital of Al-kharga District, and were subjected to Structured Clinical Interview of DSM-IIIR and Hamilton Depression Scale.

Results: Prevalence of post stroke depression (PSD) was $28.4 \%$. It was higher among males (58.4\%) than females (41.6\%). Moderate depression was the most prevalent degree among males (72\%), while severe depression was the most prevalent degree among females (57\%). Depression was more prevalent in urban (74\%) than rural communities (26\%). PSD according to the duration of stroke was more prevalent among patients after 1 year of stroke $(63.6 \%)$, followed by patients within 1 month (14.3\%). PSD was more prevalent in older age groups ( $59.7 \%$ in patients of $\geq 60$ years) followed by ( $35.1 \%$ in patients from 40 to $<60$ years age). Depression was more prevalent after ischemic stroke $(88.3 \%)$ than after Hemorrhagic $(11.7 \%)(\mathrm{P}<0.0001)$.

\section{6}

\section{Risk Factors of Stroke in Egypt}

\section{Ossama Abdulghani}

Ain Shams University, Department of Neurology and Psychiatry, Cairo, Egypt

Few epidemiologic studies are available in the Arab countries. For instance, the incidence of carotid atherosclerosis is an important risk 
factor for stroke in all western studies. However in blacks and eastern studies intracranial atherosclerosis seems to be more significant. We have established the first stroke unit in our country on 1991, and since that time we have been interested in collecting data regarding important epidemiologic facts in our country regarding stroke. It is clear that carotid atherosclerosis is much less frequent in Egyptian patients than intracranial atherosclerosis. Different profile of risk factors and genetic background are possible causes. In adults, hypertension, DM, Cardiac diseases and intracranial atherosclerosis are the most important risk factors. In younger patients, rheumatic hear disease and other cardiac causes are more important. In this Presentation, a meta-analysis of data regarding Egyptian stroke patients will be presented with special reference to potential perspective for management.

\section{7 \\ Protective Factors against Stroke Occurrence}

Valery Feigin

National Research Centre for Stroke, Applied

Neurosciences and Neurorehabilitation, Faculty of Health

and Environmental Studies, AUT University, Auckland,

New Zealand

Stroke occurrence is influenced by a complex interplay of both risk and protective factors. However, most of the research in this area has been concentrated on stroke risk factors, with far less attention paid to protective factors in relation to stroke occurrence. This presentation will be based on a literature review of some selected factors that are shown, or at least suggested, to act as protective factors against stroke occurrence, such as blood pressure and cholesterol lowering, elevated levels of HDL-C, low to moderate alcohol intake, adequate nutrition (e.g., diet rich in fish, fruits and vegetables and low in fat and sodium) and physical activity. Common strategies for stroke prevention will also be discussed.

\section{Session III. Dementia}

8

\section{Prevalence of Dementia in Al-Kharga District-New Valley (Egypt)}

Hamdy N. El Tallawya, Ahmed M. Hegazy', N. Abdel Hakeem M' , Tarek A. Rageha, Ghaydaa A. Shehata a, Noha Abo-Elfetoha, Wafaa M. Farghalya , Esam M. El Mesalhy , Ibrahim A. Rayan ${ }^{\mathrm{b}}$

aDepartment of Neurology, Faculty of Medicine, Assiut University, ${ }^{b}$ Department of Neurology, Faculty of Medicine, El-Azhar University, (Assiut branch), Assiut, Egypt

Background: Dementia is primarily a disease of the elderly. With rise in the life expectancy, there has been a parallel increase in the prevalence and incidence of dementia world wide.
Aim: To study epidemiology of dementia among the population of Al-Kharga District, New-Valley.

Methods: A 3-stages, cross sectional, door to door study (including every door) was carried out to screen 8,173 subjects (the whole population $\geq 50$ years old) out of 62,583 inhabitants in Al-Kharga District by 3 specialists of neurology, during the period from April $1^{\text {st }}$ 2005 to July $31^{\text {st }} 2008$.

A modified form of Mini- Mental State Examination (MMSE) was applied to each subject. Subjects score 23 or less (21 or less for illiterates) were investigated as probable cases of dementia. All these cases were subjected to DSM-IV-TR diagnostic criteria for dementia, full clinical assessment, psychometric assessment (Cognitive Abilities Screening Instruments (CASI), Haschinsky Ischeamic Score, Instrumental Activities Of Daily Living Scale and Geriatric Depression Scale), as well as neuro-imaging (C.T \& or MRI brain), laboratory investigations, and some specific investigations for selected patients.

Results: Dementia was recorded among 185 subjects yielding a crude prevalence rate of $2.26 \%$ for the population $\geq 50$ years. Prevalence rate increases steadily with increasing age to be $4.45 \%$ for those $\geq 60$ years old, $9.28 \%$ for those $\geq 70$ years and $18.48 \%$ for those $\geq 80$ years. Diagnosis of subtypes of dementia (164 cases) was carried out according to DSM-IV-TR criteria Into Alzheimer's disease 51.2\%, vascular dementia $28.7 \%$, dementia due to general medical conditions $12.8 \%$, dementia due to multiple etiologies $7.3 \%$. Prevalence rates for both Alzheimer's disease and vascular dementia were 1.03\%, $0.58 \%$ respectively among the population $\geq 50$ years old and $2.03 \%, 1.07 \%$ respectively among the population $\geq 60$ years old. Residence and educational level, were found to affect the prevalence of dementia.

\section{9}

\section{Alzheimer's Disease: Diagnosis and Treatment}

Jeffrey L. Cummings

UCLA Alzheimer's Disease Center, Deane F. Johnson Center for Neurotherapeutics, David Geffen School of Medicine at UCLA, Los Angeles, Calif., USA

Alzheimer's disease (AD) is a progressive neurodegenerative disease manifested by deterioration in cognition and activities of daily living and development of behavioral disturbances. AD doubles in frequency every 5 years after the age of 60 , rising from a prevalence of $1 \%$ in 60 year olds to $40 \%$ in 85 year olds. All populations of the world are experiencing an increase in the number of aged individuals in the society and a concomitant increase in the number of persons with $\mathrm{AD}$. There is a more marked increase in the proportion of aged individuals in countries with developing economies. AD typically begins with an episodic type of memory loss and progresses to include abnormalities of language, visuospatial skills, and executive functions. The disease advances to a severe state with diminished ambulation and incontinence; patients usually succumb from dementia-related infections (aspiration pneumonia and urinary tract infections). Pathologically, AD is characterized by neuritic plaques, neurofibrillary tangles, and neuron cell loss. There is increasing consensus that the production and accumulation of amyloid beta protein $(A B)$ is the initiating event of the disease. $A \beta$ accumulation in the brain appears to predate the onset of the disease by at least a decade. The symptom- 
atic portion of the disease may be more closely related to tau protein abnormalities, neurofibrillary tangles, and diverse pathways leading to cell death. Biomarkers such as MRI measures of brain atrophy, amyloid imaging, and studies of CSF $A ß$ and tau proteins reflect the evolving histopathological changes of the brain. Current therapies address the cholinergic deficit of $\mathrm{AD}$ (cholinesterase inhibitors) or produce NMDA receptor antagonism to enhance neuronal function. Many evolving therapies are aimed at decreasing production, reducing aggregation or enhancing removal of $\mathrm{A} ß$. Other novel therapies involve improving mitochondrial function, reducing tau protein aggregation, or enhancing other transmitter systems. New treatment regimens will include disease-modifying agents to slow disease progression and symptomatic treatments to improve cognitive function and behavior.

\section{Session IV. Extrapyramidal Disorders}

\section{0 \\ Epidemiology of Parkinsonism in Al-Kharga District-New Valley (Egypt)}

Hamdy N. El-Tallawya, Wafaa M. Farghalya, Noha A. Abo-Elftooha, Tarek A. Rageha, Ghaydaa A. Shehataa, Nabil A. Metwallyb

aNeurology and Psychiatry Department-Assiut University, ${ }^{\text {b }}$ Neurology and Psychiatry Department-Alazhar University (Assiut Branch), Assiut, Egypt

Background: Parkinson's disease (PD) is the second most common neurodegenerative disorder after Alzheimer's disease and is expected to impose an increasing social and economic burden on societies as the population goes older, (Lonneke, and Monique 2006).

Aim: To estimate the prevalence of Parkinson's disease and other types of parkinsonism among persons aged 40 or older in Alkharga district -New Valley.

Methods: This is a cross-sectional study, carried out through 3 stages from April $1^{\text {st }} 2005$ to July $31^{\text {st }} 2008$. Sample size of 15,482 persons aged 40 years and more, out of the total population of 62,583 persons were screened through a door to door (including every door) by three specialists of Neurology and fifteen female social workers. Positive cases were subjected to meticulous clinical, and neurological examination (using Unified Parkinson's Disease Rating Scale) by three staff members of neurology each separately. Strict diagnostic criteria were used to diagnose Parkinson's disease (Gelb et al.1999) and other types of Parkinsonism with the aid of neuro-radiological and laboratory studies, in order to minimize the impact of false positives results.

Results: We identified 49 patients of Parkinsonism with age specific ( $\geq 40$ years) prevalence rate of 316.50/100,000 (CI 95\% 240.21404.98). Out of these cases, 33 persons were diagnosed as Parkinson's disease with a prevalence rate of $213.15 / 100,000$ (CI95\% 150.51285.80), and 14 persons as vascular parkinsonism with a prevalence rate of 90.43/100,000 (CI95\% 49.60-137.78). Other types of parkinsonism (post encephalitic and unspecified) have a prevalence rate of 6.46/100,000 for each. Prevalence of Parkinsonism increases steadily with age. Also prevalence of all types of Parkinsonism appears to be statistically higher in rural than urban communities with no significant difference between men and women.

11

\section{Epidemiology of Chorea, Athetosis and Dystonia in Al-Kharga District-New Valley (Egypt)}

Hamdy N. El-Tallawya, Wafaa M. Farghalya, Tarek A. Rageha, Ghaydaa A. Shehataa, Noha A. Abo-Elftoha, Nabil A. Metwallyb, Ahmad M. Hegazy ${ }^{\mathrm{b}}$

aNeurology and Psychiatry Department-Assiut University , Neurology and Psychiatry, bDepartment-Alazhar University (Assiut Branch), Assiut, Egypt

Background: Extrapyramidal movement disorders are symptoms and signs of various disease' entities affecting different parts of the motor system. There are few published data on the prevalence of other extrapyramidal disorders in the general population compared with Parkinsonism.

Aim: To estimate the prevalence of Chorea, Athetosis and Dystonia in Al-kharga District-New Valley

Methods: This is a cross-sectional study, carried out through 3 stages from April $1^{\text {st }} 2005$ to July $31^{\text {st }} 2008$.A total population of 62,583 persons were screened through a door to door (including every door) by three specialists of Neurology and fifteen female social workers. Suggested cases of chorea, dystonia or athetosis were subjected to meticulous clinical and neurological re-evaluation by three staff members of neurology each separately in Assiut University Hospital. Patients were diagnosed as having chorea, dystonia or athetosis, when all three examiners made the same diagnosis separately. Special investigations required for diagnosis of different types of these disorders and possible etiologies were carried out in Assiut University Hospital.

Results: Twenty patients of chorea were identified with total prevalence of $31.96 / 100,000$ (95\%CI 17.95-45.96). Cerebral palsy and rheumatic chorea are the most frequent types with a prevalence of $11.85 / 100,000$ (95\%CI 2.90-19.47) and 7.99/100,000 (95\%CI 0.9914.99) respectively. Nineteen patients of dystonia were identified with a total prevalence rate of 30.36/100,000 (95\%CI 16.71-44.01). Primary dystonia represents $36.8 \%$ of the cases with a prevalence of $11.19 / 100,000$ (95\%CI 2.90-19.47), while other types of dystonia includes, cerebral palsy $(42.1 \%)$, post-encephalitic dystonia $(10.5 \%)$ and metabolic disorders $(10.5 \%)$. Only one case of athetosis was encountered with a total prevalence of 1.6/100,000 (95\%CI -1.534.73), which was diagnosed as post-encephalitic athetosis. 
12

\section{Gender Effect on Parkinson's Disease}

Giovanni Savettieri

Department of Clinical Neurosciences, University of

Palermo, Palermo, Italy

Parkinson's disease (PD) is a neurodegenerative disorder affecting the substantia nigra with loss of dopaminergic neurons. Epidemiological data has shown that PD incidence and prevalence is greater in men, suggesting a gender associated low risk for women. Clinical, epidemiologic and experimental investigations suggested that the protective effect on female is mediated by estrogen. A neurotrophic effect of estrogen on dopaminergic neurons has been indeed demonstrated in animals and in cell cultures. Analytic epidemiologic studies, has show as the risk to develop PD is associated with the length of fertile life, and the age at menopause. Also, oophorectomy performed before menopause seems to be associated with an increased risk of PD.

\section{3 \\ Clinical and Electrophysiological Assessment of Focal Dystonia}

Jun Kimura

University of lowa, lowa City, lowa, USA

Dystonia has two characteristic features distinct from other involuntary movements, sustained muscle contractions and twisting nature of abnormality. These combined induce abnormal posture, giving rise to torsional movement, as implied by the phrase, torsion dystonia. More common idiopathic type without identifiable underlying cause shows an estimated overall prevalence of 329 per million including 294 cases of focal dystonia such as blepharospasm, torticollis and writer's cramp. The underlying abnormality in torticollis involves central motor programming for head position. Task specific hand cramps develop during writing, typing and piano and guitar playing. In these patients, electromyography shows generalized muscle spasms with co-contraction of agonist and antagonist muscles, the findings characteristic of all focal dystonias

14

The Effect of Physiotherapy on Cervical Dystonia Patients Injected with Botulinum Toxin Type A

\section{Mohamed S. El-Tamawy \\ Cairo University, Cairo, Egypt}

To evaluate the effect of physiotherapy on cervical dystonia patients already injected with botulinum toxin, type A (BTX), Botox, 30 patients were included in this study; divided into 2 groups. Group
A: 15 patients compromised the study group, exposed to BTX as well as physiotherapy in the form of TENS application for 30 minutes followed by prolonged stretch for 10 minutes for each muscle, applied every other day for 8 weeks. Group B: 15 patients served as a control group, exposed to BTX injection and placebo. Assessment of both groups was done twice: at day 0 and one month after BTX injection, by using CROM, Disability Rating Scale and EMG for each muscle. It was concluded that TENS and prolonged stretch together were effective in enhancing the inhibitory effect on hyperactive muscles injected with BTX in cervical dystonia patients.

\section{Session V. Miscellaneous}

15

\section{Epidemiology of Cerebellar Ataxia in Al-Kharga District-New Valley (Egypt)}

Wafaa M. Farghaly", Hamdy N. El-Tallawy a, Tarek A. Rageha, Ghaydaa A. Shehata', Nabil A. Metwally', Noha M. AboElfetoh $^{\mathrm{a}}$

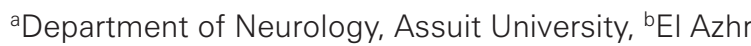
University- Assiut branch, Assiut, Egypt

Background: Rare comprehensive epidemiological studies of ataxia have been undertaken worldwide.

Aim of the Work: To estimate the prevalence of Cerebellar Ataxia, and its subtypes, in Al-Kharga District - New Valley.

Subjects and Method: This is a cross-sectional study carried out through 3 stages from April $1^{\text {st }} 2005$ to July $31^{\text {st }} 2008$.A Total populations 62,583 were screened in a door to door manner (including every door) by 3 specialists of neurology and 15 female social workers. All suspected patients with ataxia were subjected to complete medical, neurological examination, and brain CT or MRI.

Results: 24 patients of Cerebellar Ataxia were recorded in this study with a prevalence rate of 38.4/100,000(95\%CI; 23.01-53.69). The order of frequency of different types of Cerebellar Ataxia was as follows; 8 patients(12.8/100,000; 95\%CI: 3.93-21.64) had vascular cerebellar ataxia, 6 patients $(9.6 / 100,000 ; 95 \% \mathrm{CI}: 1.92-17.26)$ had ataxic cerebral palsy, 5 (8/100,000; 95\%CI: 0.99-14.99) had unclassified progressive cerebellar ataxia (?metabolic), 3(4.8/100000; 95\%CI: $0.63-10.22)$ had post encephalitic ataxia and 2(3.2/100,000; 95\%CI: 1.23-7.62) had Fredriche's ataxia. 


\section{6}

\section{Epidemiology of Cerebral Palsy in Al-Kharga District-New Valley (Egypt)}

\author{
Hamdy N. El-Tallawy', Wafaa M. Farghalya, Ghaydaa A. \\ Shehat ${ }^{a}$ Nabil A. Metwally ${ }^{\mathrm{b}}$, Tarek A. Rageh ${ }^{\mathrm{a}}$,Noha M. \\ Abo-Elfetoh ${ }^{\mathrm{a}}$ \\ aDepartment of Neurology, Assuit University and bEl Azhr \\ University- Assuit Branch, Assiut, Egypt
}

Background: Cerebral Palsy (CP) comprises a group of permanent and non-progressive disorders of movement.

Aim of the Work: To estimate the prevalence, subtypes and incidence of Cerebral Palsy in Al -Kharga District -New Valley.

Subjects and Method: This is a cross sectional study carried out through 3 stages from April $1^{\text {st }} 2005$ to July $31^{\text {st }} 2008$.All populations $(62,583)$ were screened in a door to door manner (including every door) by 3 specialists of neurology and 15 female social workers. Each child with suspected CP was subjected to complete medical history, clinical examination, brain MRI, EEG and Stanford-Binet ( $4^{\text {th }}$ edition).

Results: This study revealed 52 children with CP out of 25,540 children $(<18$ years). This yielded an age specific prevalence rate of CP 2.03/1000 (95\%CI: 1.48-2.59) of living birth. Mean age of patients with $\mathrm{CP}$ was $7.17 \pm 4.38$ years. The order of frequency of different subtypes of $\mathrm{CP}$ was as follows; 34 patients $(65.4 \%)$ had spastic type of CP, $14(26.9 \%)$ had mixed type and $2(3.8 \%)$ for each ataxic and dyskintic types of CP. The annual incidence of CP in last 18 years showed a steady increase from $0.75 / 1000$ at 1990 to its maximum value $3.6 / 1000$ at 2004 . Then it declined to $2.4 / 1000$ in the last 3 years (2005-2007). This was true for male patients with CP, as well as those in urban or rural communities. While this trend of incidence among females showed a steady increase allover the studied period from $0.8 / 1000$ at 1990 to $3.9 / 1000$ at 2007 .

\section{7}

\section{Epidemiology of Bell's Palsy in Al-Kharga District-New Valley (Egypt)}

Hamdy N. El. Tallawy', Wafaa M. Farghalya, Noha M.

Abo-Elfetoh', Tarek A. Rageha, Nabil A. Metwally',

Ghaydaa A. Shehata ${ }^{a}$

aDepartment of Neurology, Assiut University, ${ }^{\text {bD Department }}$ of Neurology, El Azhar University, Assiut branch, Assiut, Egypt

Background: Bell's Palsy (BP) is a relatively common disease of unclear etiology characterized by sudden onset of unilateral facial paralysis. Data regarding the frequency of BP are limited and contradictory in epidemiological findings especially in developing countries.

Aim: to determine the epidemiology of BP in Al -Kharga District -New Valley.

Subject and Methods: A cross section study carried out through 3 stages from April $1^{\text {st }} 2005$ - July $31^{\text {st }} 2008$. A Total population of 62,583 was screened through a door-to-door survey (every door) by three specialists of Neurology and 15 female social workers.
All subjects who had experienced facial paralysis through their lifetime were subjected to meticulous neurological examination by a specialist of Neurology to exclude facial paralysis related to other causes.

Results: Among population aged 8 years and above, 100 patients who were diagnosed to have BP were subjected to specific questionnaire for BP. The lifetime prevalence rate was 192.18 / 100,000 (95\% CI 155-230). Age and sex specific lifetime prevalence rate was higher among women than men [245.81 and 141.73 / 100,000 respectively], with odds ratio for sex 1.74 [95\% CI for risk (1.16-2.60)]. It showed a significant increase with age, with a peak prevalence rate at middle adulthood, and a significantly $(\mathrm{p}<.002)$ higher prevalence rate among rural than urban inhabitants. Fifty four cases of BP occurred within the last two years of our study, with an incidence rate within these last two years of 108.24 per 100,000 population ( $\geq 8$ years) [ $95 \%$ CI (79137)]. The incident cases were higher in the second year than the first year (37 and 17 respectively).

\section{8 \\ Epidemiology of Muscle and Neuromuscular Disorders in Al-Kharga District-New Valley (Egypt)}

Wafaa M. Farghalya, Hamdy N.A. El-Tallawya, Ghaydaa A. Shehata', Tarek A. Rageha, Noha Abo El Fotooh', Nabiel Abdel Hakeem ${ }^{\text {b }}$

aDepartments of Neurology and Psychiatry, Assiut University, ${ }^{\text {b} A l a z h a r ~ U n i v e r s i t y, ~ A s s i u t ~ B r a n c h, ~ A s s i u t, ~}$ Egypt

Background: Muscle diseases have become the subject of extensive research with regard to their classification, molecular pathology and inheritance, but relatively few epidemiological studies have been undertaken (Gautier, and Fardeau, 1995).

Aim: To estimate the prevalence of Muscle and Neuromuscular Disorders in Al-Kharga District, New Valley.

Methods: This is a cross-sectional study conducted in Al-Kharga District, through a door-to-door survey (including every door) from April 1, 2005 to July 31, 2008. Patients identified from the survey as probable muscle or neuromuscular disorder, were subjected to full clinical examination, with confirmation of the diagnosis through electrophysiological study, and laboratory investigations (CPK).

Results: Twenty patients with muscle disorders were identified, with a total prevalence rate of $32 / 100,000$ with insignificant difference between rural $7(38.9 / 100,000)$ and urban $13(29.2 / 100,000)$ communities. No cases of Myotonia, Myositis or Secondary myopathies were recorded. The age specific $(0-\leq 30$ years $)$ prevalence was $50 / 100,000(46.2 / 100,000$ for urban population and 59.1/100,000 for rural population). The highest prevalence $(30 / 100,000)$, was recorded for Duchenne muscular dystrophy with a significantly higher prevalence among urban than rural community. Other types of Muscle disorders were; Limb Girdle Myopathy (pelvic type) 3 patients (7.5/100,000), Childhood Myopathy 2 female patients $(5 / 100,000)$, Congenital Myopathy 1 female patient $(2.5 / 100,000)$, Mitochondrial Myopathy (MERRF) 2 patients $(5 / 100,000)$. Two female patients were diagnosed to have Myasthenia Gravis with a prevalence rate of $(5 / 100,000)$. 


\section{9}

\section{Epidemiology of Primary Nocturnal Enuresis in Al-Kharga District-New Valley (Egypt)}

Wafa M.A. Farghalya, Hamdy N.A. El-Tallawya, Nabiel Abde I Hakeem ${ }^{\mathrm{b}}$, Noha Abo El Fotooha, Ghydaa A. Shehata,

Tarek A. Rageh ${ }^{\text {a }}$

Departments of Neurology and Psychiatry, ${ }^{\text {aAssiut }}$ University, ${ }^{b}$ Alazhar University, Assiut Branch, Assiut, Egypt

Background: Nocturnal Enuresis (NE) is one of the most frequent pediatric pathologies and can have a profound effect on children and their families (Lottmann, and Alova; 2007).

Aim: To estimate the prevalence of primary NE among children in Al-Kharga District.

Method: The present cross-sectional study was conducted in Alkharga District (New Valley), through a door-to-door survey (including every door) from April $1^{\text {st }} 2005$ to July $31^{\text {st }} 2008$. A total 17,770 children aged from 6 to $<18$ years old were included in the study. Identified patients were subjected to detailed history taking, and a full clinical examination. Cases identified with epilepsy, mental retardation, and spina bifida (as diagnosed by plain X-ray) were excluded.

Results: Three hundred forty two patients were diagnosed to have primary NE in this study, with an age specific prevalence $(6-18$ year) rate of $1.92 \%$. There was a significant higher percent of NE among urban than rural in total community, and among all age groups except the age from 15 to 18 years. Two hundreds thirty one patients with primary NE aged 6 to 12 years old (67.5\%). Family history of NE was recorded among 198 patients $(57.9 \%)\{21.9 \%$ among sisters and, $16.7 \%$ among brothers $\}$. Two hundreds $(58.5 \%)$ of the patients with primary NE were noticed by their parents as heavy sleepers. Psychological stress was recoded among 165 (48.2\%) patients \{severe punchment $(18.4 \%)$ followed by birth of a second child (12.6\%)\}, and $38(11.1 \%)$ patients had more than one stressors. Most of children $(89.5 \%)$ wet their bed daily or every other day. Some foods may contribute to NE.

\section{0}

\section{CNS Drug Discovery}

\section{Jeffrey L. Cummings}

UCLA Alzheimer's Disease Cente, Deane F. Johnson Center for Neurotherapeutics, David Geffen School of Medicine at UCLA, Los Angeles, Calif., USA

Neurology is entering a remarkable period of growth and transition. Advances in understanding the basic pathophysiology if brain diseases is resulting in the development and testing of new drugs the will control the symptoms, slow disease progression, and improve the quality of life of patients with neurologic disease and their families and caregivers. Neurology is transitioning from a specialty with primarily symptom-based treatments into an era of disease-modifying treatment. Drug discovery and development is an arduous process with few predictors of success (e.g., predictors of efficacy or of side effects that will limit development). Most compounds that enter the development pipeline fail to make it to market; it is currently esti- mated that of 100 compounds that enter Phase I (first in human testing) only 6 will be advanced to the regulatory authorities for marketing access. Candidate treatments are increasingly focused on targets within disease cascades that lead to neurodegeneration, inflammation, neoplasia, or episodic events (seizures, migraine). Candidate therapies may be discovered by high-throughput screening of compound libraries, computerized (in silico) design, by modifying or repurposing existing drugs, or through study of diet, biodiversity or traditional medications. Agents that are promising in screens are assessed for "druggability" - likely toxicity, preliminary pharmacokinetics, ability to cross the blood brain barrier. From this stage, agents that are druggable are tested in preclinical studies (laboratory animals) for toxicity and pharmacokinetics and in disease models for efficacy (e.g., transgenic species). Compounds with promising efficacy and acceptable tolerability are advanced to Phase I first in human studies. Participants are typically young healthy volunteers. Single dose studies are followed by multiple dose studies lasting up to 14 days. The pharmacokinetics of the agent in humans --- half-life, active metabolites, route of metabolism, excretion --- are determined. Safety and tolerability are evaluated. If the agent appears to be safe and tolerable, it is advanced to Phase II. These studies provide proof-of-concept (POC) in the specific disease state and determine the doses that are to be advanced to Phase III. Phase II involves small numbers of patients with the target disease. Once POC is achieved and doses are determined, the drug can be advanced to Phase III pivotal trials. These trials are designed to assess the safety and efficacy of the agent in large populations of patients with the target disease. Success at this stage is followed by submission to the regulatory authorities for permission to market. The entire process requires 10-14 years and costs approximately $\$ 600$ million USD. So far the yield of this rational discovery and development process has been small. Improved understanding of disease mechanisms and target validation along with improvements in the drug development process, promise to produce more effective agents that will affect fundamental disease mechanisms and enhance the lives of patients with neurologic diseases.

21

\section{Epidemiology of MS in Egypt}

Saher Hashem, S.H. Hebah

Cairo University, Cairo, Egypt

Multiple sclerosis, previously called disseminated sclerosis in Egypt is not a recent discovery. The first ever known work on DS,IN EGYPT dates back to 1954 when Prof. DR MAHMOUD MOSTAFA issued his doctor degree in neurology using some primitive invasive investigations to exclude MS like myelography and angiography, giving prevalence of $3.7 \%$. Thus the diagnosis was through exclusion and not typicality of the disease. In 1960"s Prof. Yahia Taher in Cairo and Prof. Elgarem in Alexandria studied the prevalence of the disease using outpatient clinics and gave prevalence of $1.3 \%$ and $0.3 \%$ respectively.After that and after the advent of MRI many studies on the prevalence in different governorates and different countries in Cairo and cosmopolitan areas wre carried out (In Cairo; Saher Hashem, Alexandria ,Farouk Talaat; Tanta, El Senosi, Assuit; Hamdy El Tellawy 2002). Many studies on different aspect of MS diagnostic and therapeutic will be presented up to year 2008 . 


\section{2}

\section{Neuroepidemiology in Sharkia Governorate}

Sami El-Shazli

Department of Neurology, Zagazig University, Sharkia, Egypt

Ten neurological studies were done in Sharkia Governorate in the period from 1987 to 2006 to evaluate the prevalence, demographic and clinical data of certain neurological diseases.

Poliomyelitis: this study was done in 1987 among school children. The residual poliomyelitis was $3.3 / 1000$. It was $2.7 / 1000$ in urban while it was 4.1/1000 among rural districts.

Epilepsy: this study was done in 1996 among school children. The prevalence rate was $8.3 / 1000$. Generalized seizures $(55.3 \%)$ was more than partial $(33.7 \%)$, males $(8.3 / 1000)$ more than females (7.9/1000).

Chronic headache: this study was done in 2000. The prevalence rate was $0.65 \%$ in males versus $0.56 \%$ in females, it was $0.71 \%$ in rural versus $0.49 \%$ in urban districts. Migraine was the most frequent $(0.48 \%)$ followed by tension headache $(0.08 \%)$ then mixed headache $(0.05 \%)$.

Cerebrovascular diseases (study 1): a field study was done in a rural district from Feb. to Jul. 1991. The prevalence rate was $91.01 / 100,000$. The incidence increased with age (27.2\%) among age group 60-69 years, males are more affected than females $(51.35 \%$ and $48.65 \%$ respectively).

Cerebrovascular diseases (study 2): the study was done on hospitalized patients in the period from 1999 to 2001, males were more affected than females among all stroke patients with much higher difference among hemorrhagic stroke patients. Incidence of stroke was higher among the age group 55-69 years, ischemic stroke was higher among age group 65-74 years, while hemorrhagic stroke among age group pf 25-44 years.

Parkinsonism: a door to door study was done in 1991. The prevalence rate was $13 / 100,000$. Paralysis agitans prevalence rate was $47 / 100,000$ in population over 40 years.

Tetanus neonatorum: 100 neonatal tetanus and 100 healthy newborns were studied. Case fatality rate was $75 \%$ which inversely related to age, the occurrence and severity of the disease were more in rural district and those neonates from non-immunized mothers.

Dementia after retirement: 3 year period (2002-2005) hospital based 3 phase study was done. The prevalence rate for specific subtypes was $1.4 \%$ for $\mathrm{AD}, 1.05 \%$ for $\mathrm{VD}, 0.5 \%$ for $\mathrm{MD}$ and $0.44 \%$ for other dementias. Age specific prevalence tends to be doubled every 5 years, with no gender difference, but it is more among illiterate patients.

Neurological disease among children: house to house study in rural district among children below age 15 years was done in 1990 , the prevalence ratio was $6.8 / 1000$, males were affected more than females (66\% and $34 \%$ respectively).

Viral encephalitis: Selected 40 patients with a primary diagnosis of viral encephalitis (HSV, CMV and rubella) children was the most affected age group $77.5 \%$, males were more affected than females (57.5\% and $42.5 \%$ respectively), rural $(87.5 \%)$ were more than urban $(12.5 \%)$.

\section{3}

\section{Differential Diagnosis of Facial Weakness}

Jun Kimura

University of lowa, lowa City, lowa, USA

Weakness of the orbicularis oculi and frontalis usually suggests a peripheral type of facial palsy, which may herald other symptoms of multiple sclerosis, systemic infection such as Lyme borreliosis and AIDS. Patients with Guillain Barre Syndrome may develop bilateral, or rarely unilateral, demyelinative conduction block. An acoustic neuroma may compress the facial nerve. Bell's palsy develops sporadically in an isolated incidence, approximately $80 \%$ showing a good prognosis with demyelination, and the rest axonal degeneration as in herpes zoster infection, which carries a less favorable outcome. The blink reflex reveals various degrees of abnormality in most of these patients. EMG shows physiologically small motor unit potentials, which may mimic fibrillation potentials.

\section{4 \\ Recent Topics in the Stroke Rehabilitation Strategy Applying Transcranial Magnetic Stimulation}

Tatsuya Mima, Satoko Koganemaru, Mohamed Nasr-Eldin Thabit, Hidenao Fukuyama

Kyoto University Graduate School of Medicine, Kyoto, Japan

Transcranial magnetic stimulation (TMS) is a powerful tool to change the brain function (neural plasticity). The change induced by TMS can remain beyond the end of TMS intervention, which may share the similar mechanism as long-term potentiation/depression. Couples of TMS protocols have been proposed for stroke patients, including the high-frequency repetitive TMS at the affected hemisphere, low-frequency repetitive TMS at the non-affected side, or other stimulation protocol such as theta-burst or paired-associative stimulation. We will discuss the theoretical basis of these strategies and introduce our recent project for stroke rehabilitation. 